\title{
Nordamnacanthal induced apoptosis and mitotic-G2/M arrest with downregulation of Bcl-2 in the human breast cancer cell line (MCF-7).
}

\begin{abstract}
Nordamnacanthal, an anthraquinone extracted from the root of Morindaelliptica from Rubiaceae family has cytotoxic properties towards cancer cell lines and anti tumor promoting activities. This study was conducted to determine the cytotoxic effect of nordamnacanthal towards MOLT-4 and MCF-7 cell lines. Nordamnacanthal was found to be more cytotoxic towards MOLT-4 than MCF-7 with the IC50 of $3.8 \mu \mathrm{g} / \mathrm{ml}$ and $54 \mu \mathrm{g} / \mathrm{ml}$, respectively, as detected by using the trypan blue dye exclusion test. The nordamnacanthal-treated cells showed characteristics of apoptosis such as membrane blebbing, chromatin condensation and formation of apoptotic bodies as observed under an inverted lightmicroscope. Fluorescence analysis of cell death using acridine orange and propidium iodide staining showed that the population of MOLT-4 and MCF-7 cells underwent apoptosis at the IC50 value was 32\% and $30.4 \%$,respectively. Cell cycle analysis by flow cytometry indicated that nordamnacanthal did arrest MCF-7 cells at the G2/M phase. For MOLT-4, no cell cycle arrest was observed. Bcl-2 and Bax were downregulated in nordamnacanthal-treated MCF-7 cells. On the other hand, expression of the proteins in MOLT-4 was not from the control. In conclusion, nordamnacanthal was more cytotoxic towards MOLT-4 than MCF-7 cell line. The compound induced apoptosis in both cell lines, but with G2/M arrest and the involvement of Bcl-2 and Bax only in MCF-7.
\end{abstract}

Keyword: Nordamnacanthal; Cytotoxicity; Apoptosis; G2/M arrest; Bcl-2; Bax. 